\title{
Activation of Mst1 causes dilated cardiomyopathy by stimulating apoptosis without compensatory ventricular myocyte hypertrophy
}

\author{
Shimako Yamamoto, ${ }^{1}$ Guiping Yang, ${ }^{1}$ Daniela Zablocki, ${ }^{1}$ Jing Liu, ${ }^{1}$ Chull Hong, ${ }^{1}$ \\ Song-Jung Kim, ${ }^{1}$ Sandra Soler, ${ }^{1}$ Mari Odashima, ${ }^{1}$ Jill Thaisz, ${ }^{1}$ Ghassan Yehia, ${ }^{2}$ \\ Carlos A. Molina, ${ }^{2}$ Atsuko Yatani, ${ }^{1}$ Dorothy E. Vatner, ${ }^{1}$ Stephen F. Vatner, ${ }^{1}$ \\ and Junichi Sadoshima ${ }^{1}$
}
${ }^{1}$ Cardiovascular Research Institute, Department of Cell Biology and Molecular Medicine, and Department of Medicine, and ${ }^{2}$ Department of Gynecology, Obstetrics and Women's Health, University of Medicine \& Dentistry of New Jersey,
New Jersey Medical School, Newark, New Jersey, USA

\begin{abstract}
Activation of mammalian sterile 20-like kinase 1 (Mst1) by genotoxic compounds is known to stimulate apoptosis in some cell types. The importance of Mst 1 in cell death caused by clinically relevant pathologic stimuli is unknown, however. In this study, we show that Mst 1 is a prominent myelin basic protein kinase activated by proapoptotic stimuli in cardiac myocytes and that Mst 1 causes cardiac myocyte apoptosis in vitro in a kinase activity-dependent manner. In vivo, cardiac-specific overexpression of Mst1 in transgenic mice results in activation of caspases, increased apoptosis, and dilated cardiomyopathy. Surprisingly, however, Mst1 prevents compensatory cardiac myocyte elongation or hypertrophy despite increased wall stress, thereby obscuring the use of the Frank-Starling mechanism, a fundamental mechanism by which the heart maintains cardiac output in response to increased mechanical load at the single myocyte level. Furthermore, Mst1 is activated by ischemia/reperfusion in the mouse heart in vivo. Suppression of endogenous Mst 1 by cardiac-specific overexpression of dominantnegative Mst 1 in transgenic mice prevents myocyte death by pathologic insults. These results show that Mst1 works as both an essential initiator of apoptosis and an inhibitor of hypertrophy in cardiac myocytes, resulting in a previously unrecognized form of cardiomyopathy.
\end{abstract}

J. Clin. Invest. 111:1463-1474 (2003). doi:10.1172/JCI200317459.

\section{Introduction}

A number of studies in the past decade have shown that cardiac myocyte death characteristic of apoptosis occurs in response to ischemia/reperfusion $(\mathrm{I} / \mathrm{R})$ and during cardiac remodeling after myocardial infarction, and is the pathogenesis of cardiomyopathy and heart failure (1-9). Although controversy still exists regarding the

Received for publication November 22, 2002, and accepted in revised form February 27, 2003.

Address correspondence to: Junichi Sadoshima, Cardiovascular Research Institute, University of Medicine \& Dentistry of New Jersey, New Jersey Medical School, 185 South Orange Avenue, MSB G-609, Newark, New Jersey 07103, USA.

Phone: (973) 972-8619; Fax: (973) 972-8919;

E-mail: Sadoshju@umdnj.edu.

Conflict of interest: The authors have declared that no conflict of interest exists.

Nonstandard abbreviations used: mammalian sterile 20-like kinase 1 (Mst1); sterile 20-like (STE 20-like) kinase; adenovirus harboring wild-type Mst1 (AdX-Mst1); adenovirus harboring dominant-negative Mst1 (K59R), (AdX-DN-Mst1); myelin basic protein (MBP); transgenic Mst1 mice (Tg-Mst1); dominantnegative Mst1 transgenic mice (Tg-DN-Mst1); left ventricle (LV); anterior descending branch of the left coronary artery (LAD), area at risk (AAR); protein phosphatase 2A (PP-2A); LV end-diastolic dimension (LVEDD) ; LV end-systolic dimension (LVESD); LV ejection fraction (LVEF); LV fractional shortening (\%FS). occurrence of apoptosis versus oncosis in the ischemic heart $(10,11)$, it seems established that the signaling mechanisms promoting myocyte cell death, including caspases, are activated by $\mathrm{I} / \mathrm{R}$ and in cardiomyopathy (12-16) (reviewed in ref. 17). Recent evidence suggests that myocyte proliferation could take place in response to the loss of cardiac myocytes after myocardial infarction (18). Considering the limited capacity of terminally differentiated cardiac myocytes for proliferation, however, it is important to understand how these cell death-promoting signaling mechanisms are activated by $\mathrm{I} / \mathrm{R}$ and in myopathic hearts in order to establish interventions that could effectively prevent the cell loss in heart disease (10). It has been shown that intracellular stress-responsive protein kinases (SRPKs), including JNKs and p38-MAPKs, are activated by I/R in the heart (19-24). Although these kinases are likely to affect both cell death and cell survival, the roles of SRPKs in cardiac myocyte apoptosis are not fully understood. Furthermore, the SRPK selectively promoting cardiac myocyte apoptosis has not been identified in vivo.

Mammalian sterile 20-like kinase 1 (Mst-1) is a ubiquitously expressed serine/threonine kinase $(25,26)$, which belongs to a mammalian sterile 20-like (STE 20like) kinase family consisting of Pak1, Mst1, Mst2, 
Khs, Gck, Sok1, Nik, Hpk1, and Sps1 $(27,28)$. Increasing lines of evidence suggest that Mst1 and other STE20-like family kinases play an important role in mediating apoptosis (reviewed in ref. 28). Mst 1 is activated by some proapoptotic stimuli in fibroblastic and lymphocytic cell lines. However, stimuli shown to activate Mst1 are generally limited to nonphysiological stresses, including genotoxic compounds and extreme heat shock $(26,29-32)$. Mst 1 can be an efficient mediator of apoptosis because it is cleaved by caspases, and this cleavage increases kinase activities of Mst1, which in turn activates caspase-3 (33), thereby constituting a powerful amplification loop of apoptotic responses. Importantly, however, whether or not activation of Mst1 is required for in vivo cell death in response to clinically relevant pathologic insults has not been determined in any organs, including the heart. Furthermore, it is unknown if Mst1 mediates cellular functions other than apoptosis.

In this study, by using both cultured cardiac myocytes in vitro and transgenic mouse models in vivo, we examined if Mst1 is activated by clinically relevant pathologic stimuli in the heart, and if so, whether or not activation of Mst 1 is sufficient to induce apoptosis in cardiac myocytes. Furthermore, we examined if activation of endogenous Mst1 is required for cardiac myocyte apoptosis by I/R. Our results indicate that Mst1 is an important mediator of cardiac myocyte apoptosis both in vitro and in vivo. Surprisingly, Mst1 also prevents cardiac myocyte elongation and hypertrophy from taking place despite increased wall stress, which potentially makes operation of the Frank-Starling mechanism less efficient at the myocyte level. We therefore propose that Mst1 could be an important therapeutic target in ischemic heart disease, cardiomyopathy, and heart failure.

\section{Methods}

Primary culture of neonatal rat ventricular myocytes. Primary cultures of ventricular cardiac myocytes were prepared from 1-day-old Crl: (WI) BR-Wistar rats (Charles River Laboratories, Wilmington, Massachusetts, USA) as described previously (34). A cardiac myocyte-rich fraction was obtained by centrifugation through a discontinuous Percoll gradient as described (34). We obtained myocyte cultures in which more than $95 \%$ were myocytes, as assessed by immunofluorescence staining with a mAb against sarcomeric myosin (MF20). Hypoxia/reoxygenation was applied to cardiac myocytes using an anaerobic chamber (Thermaquest Forma Scientific Division, Marietta, Ohio, USA) at $37^{\circ} \mathrm{C}$.

Construction of the adenoviral vectors. We constructed recombinant adenovirus using an Adeno-X adenovirus construction kit (CLONTECH Laboratories Inc., Palo Alto, California, USA). We made replicationdefective human adenovirus type 5 (devoid of $\mathrm{E} 1$ and E3) harboring wild-type Mst1 (AdX-Mst1) and dominant-negative Mst1 [Mst1 (K59R)] (AdX-DN-Mst1).
Adenovirus harboring $\beta$-galactosidase (Ad5 $\beta$ gal) was used as a control. Generation of adenovirus harboring XIAP (Ad5 XIAP) has been described (35).

Analysis of DNA fragmentation by ELISA and DNA-laddering assays. Histone-associated DNA fragments were quantified by the Cell Death Detection ELISA (Roche Molecular Biochemicals, Indianapolis, Indiana, USA) as described (35), with minor modification for sample preparation. In brief, myocytes were rinsed with PBS three times in order to remove nucleosomes leaked out from necrotic cells and then incubated with the lysis buffer for $30 \mathrm{~min}$. The supernatant containing monoand oligo-nucleosomes from the cytoplasmic fraction of apoptotic cells was used for further analyses. DNA fragmentation of the tissue samples was determined by DNA-laddering assays as described (36).

Immunoblot analysis. For analyses of Mst1, cells were lysed in lysis buffer A containing $25 \mathrm{mmol} / 1 \mathrm{NaCl}, 25$ $\mathrm{mmol} / \mathrm{l}$ Tris ( $\mathrm{pH} 7.4$ ), $1 \mathrm{mmol} / \mathrm{l} \mathrm{Na} \mathrm{VO}_{4}, 10 \mathrm{mmol} / \mathrm{l} \mathrm{NaF}$, $10 \mathrm{mmol}$ sodium pyrophosphate, $0.5 \mathrm{mmol} / \mathrm{l}$ EGTA, 0.5 $\mathrm{mmol} / \mathrm{l}$ AEBSF, $0.5 \mu \mathrm{g} / \mathrm{ml}$ leupeptin, and $0.5 \mu \mathrm{g} / \mathrm{ml}$ aprotinin. For immunoblotting, we used anti-human Mst $1 \mathrm{mAb}$ (Transduction Laboratories, Lexington, Kentucky, USA) for detection of the carboxyl terminus of Mst1. Rabbit polyclonal Ab was raised against animoterminal human Mst1 ETVQLRNPPRRQLKC (pAb-15) (BioSource International, Camarillo, California, USA) and affinity purified for detection of the amino-terminus of Mst1. Polyclonal Ab's raised against cleaved caspase-3 (Cell Signaling Technology Inc., Beverly, Massachusetts, USA) were used to determine activation of caspase-3. Preparation of mitochondrial-free cytosolic fraction has been described (37). Anti-cytochrome c AB (Pharmingen, San Diego, California, USA) was used for immunoblot of cytochrome. Phosphorylation of p38MAPK and p46-JNKs was determined as described (38).

In-gel kinase assay. In-gel myelin basic protein (MBP) kinase assays were performed as described previously (39). Either $100 \mu \mathrm{g}$ of total cell lysates, heart homogenates, or immunoprecipitates of Mst1were used. Immunoprecipitation of Mst1 was performed by incubating cell extracts $(300 \mu \mathrm{g})$ with $\mathrm{pAb}-15$ at $4^{\circ} \mathrm{C}$ for $3 \mathrm{~h}$ followed by $40-\mu \mathrm{l}$ slurry $(50 \% \mathrm{vol} / \mathrm{vol})$ of protein A-Sepharose at $4^{\circ} \mathrm{C}$ for $1 \mathrm{~h}$. The samples were washed with lysis buffer $(700 \mu l)$ three times.

Transgenic mice. Mst1 and dominant-negative Mst1 (DN-Mst1) transgenic mice (hereafter designated as TgMst1 and Tg-DN-Mst1, respectively) were generated (C57BL/6 background) using a cDNA of human mycMst1 and myc-Mst1 (K59R) driven by the $\alpha$-myosin heavy chain promoter (courtesy of J. Robbins, University of Cincinnati, Cincinnati, Ohio, USA) to achieve cardiac-specific expression.

Echocardiography. Mice were anesthetized with an intraperitoneal injection of ketamine $(0.065 \mathrm{mg} / \mathrm{g})$, acepromazine $(0.02 \mathrm{mg} / \mathrm{g})$, and xylazine $(0.013 \mathrm{mg} / \mathrm{g})$. Echocardiography was performed using ultrasonography (Apogee CX-200; Interspec Inc., Ambler, Pennsylvania, USA) as described previously (38). 
Histological analyses. The heart specimens were fixed with formalin, embedded in paraffin, and sectioned at $6-\mu \mathrm{m}$ thickness. Interstitial fibrosis was evaluated by picric acid Sirius red staining as described (40). Myocyte cross-sectional area was measured from images captured from silver-stained 1 - $\mu \mathrm{m}$-thick methacrylate sections as described $(36,38,40)$. Suitable cross sections were defined as having nearly circular capillary profiles and circular-to-oval myocyte sections. No correction for oblique sectioning was made. The outline of 100-200 myocytes was traced in each section. The MetaMorph image system software was used to determine myocyte cross-sectional area $(36,38,40)$. The number of myocyte $(N)$ in the sampled area $(A)$ was measured according to the criteria described by Gundersen (41) using Image-Pro Plus software, and the myocyte density was calculated as $N / A$. Using the histologically determined thickness of the left ventricle (LV) free wall and the myocyte density per unit area of myocardium, the average number of myocytes across the ventricular wall was computed as described (42). Evaluation of apoptosis in tissue sections. DNA fragmentation was detected in situ using TUNEL, as described $(36,38)$. Briefly, deparaffinized sections were incubated with proteinase $\mathrm{K}$, and DNA fragments were labeled with fluorescein-conjugated dUTP using TdT (Roche Molecular Biochemicals). Nuclear density was determined by manual counting of DAPI-stained nuclei in six fields of each animal using the $40 \times$ objective and the number of TUNEL-positive nuclei counted by examining the entire section using the same power objective. Limiting the counting of total nuclei and the TUNELpositive nuclei to areas with a true cross section of myocytes made it possible to selectively count only those nuclei that clearly were within myocytes (36).

Isolation of adult mouse cardiac myocytes. Cardiac myocytes were isolated as described previously (43). Longitudinal length of ventricular cardiac myocytes was determined as described (43). Whole cell currents were recorded using patch-clamp techniques (44). Cell capacitance was measured using voltage ramps of 0.8 $\mathrm{V} / \mathrm{s}$ from a holding potential of $-50 \mathrm{mV}$.

$I / R$ surgery in vivo. Mice were anesthetized by intraperitoneal injection of pentobarbital sodium $(60$ $\mathrm{mg} / \mathrm{kg}$ ). A rodent ventilator (model 683; Harvard Apparatus Inc., Holliston, Massachusetts, USA) was used with $65 \%$ oxygen during the surgical procedure. The animals were kept warm using heat lamps and heating pads. Rectal temperature was monitored and maintained between 36.8 and $37.2^{\circ} \mathrm{C}$. The chest was opened by a horizontal incision through the muscle between the ribs (third intercostal space). Ischemia was achieved by ligating the anterior descending branch of the left coronary artery (LAD) using a 8-0 nylon suture, with a silicon tubing ( $1 \mathrm{~mm}$ OD) placed on top of the LAD, $2 \mathrm{~mm}$ below the border between left atrium and LV. Regional ischemia was confirmed by ECG change (ST elevation). After occlusion for $20 \mathrm{~min}$, the silicon tubing was removed to achieve reperfusion.
The chest wall was closed by with $8-0$ silk. The animal was removed from the ventilator and kept warm in the cage maintained at $37^{\circ} \mathrm{C}$ overnight. Hearts were harvested after $24 \mathrm{~h}$ of reperfusion.

Assessment of area at risk and infarct size. After I/R, the animals were reanesthetized, intubated, and chest was opened. After arresting the heart at the diastolic phase by $\mathrm{KCl}$ injection, the ascending aorta was canulated and perfused with saline to wash out blood. The LAD was occluded with the same suture, which had been left at the site of the ligation. To demarcate the ischemic area at risk (AAR), Alcian blue dye (1\%) was perfused into the aorta and coronary arteries. Hearts were excised, and LVs were sliced into $1-\mathrm{mm}$

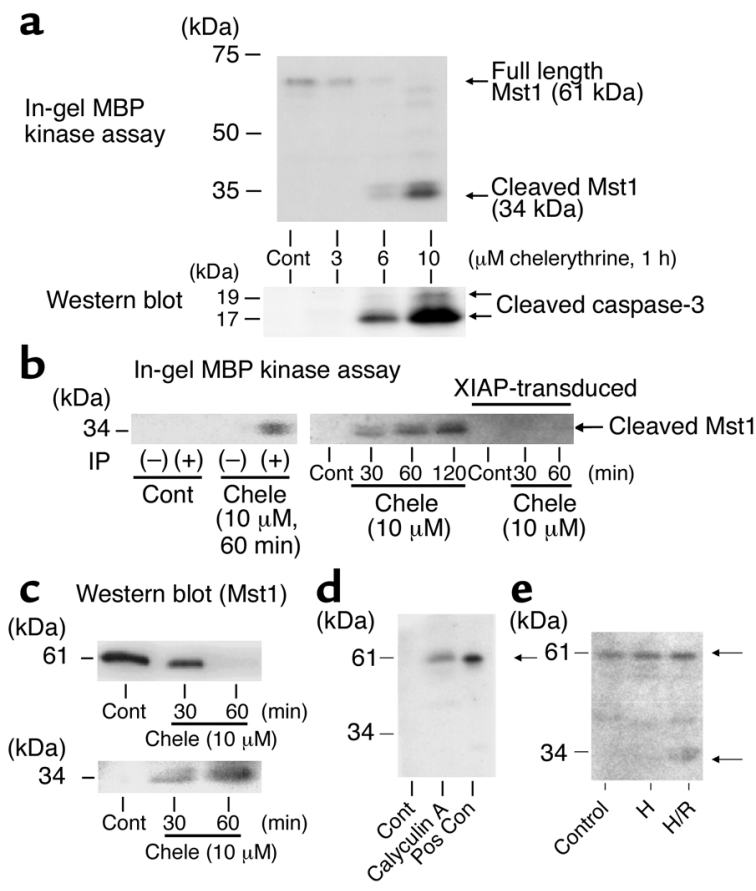

\section{Figure 1}

(a) Cardiac myocytes were treated with indicated concentrations of chelerythrine for $1 \mathrm{~h}$. Cell lysates were subjected to in-gel MBP kinase assay as well as immunoblotting with anti-cleaved caspase-3 Ab. Results are representative of more than five experiments. (b) Cardiac myocytes were treated with chelerythrine (Chele). In the right panel, myocytes were transduced with either control virus or adenovirus harboring XIAP 48 h before Chele application. Cell lysates were subjected to immune complex in-gel MBP kinase assays, using anti-Mst1 polyclonal Ab (pAb-15). The activity of cleaved Mst 1 is shown. $n=3$. (c) Cardiac myocytes were treated with Chele for the indicated durations. Immunoblot analyses were conducted using anti-Mst1 mAb (upper panel) and anti-Mst1 polyclonal Ab (lower panel), which detects the full-length form and cleaved form (amino-terminal half) of Mst1, respectively. $n=5$. (d) Cardiac myocytes were treated with vehicle (lane 1), calyculin A ( $1 \mu \mathrm{M}$, lane 2 ), or adenovirus harboring Mst1 (10 MOI) as positive control (Pos Con, lane 3). In-gel MBP kinase assays were performed. An arrow indicates the full-length form of Mst1. $n=3$. (e) Cardiac myocytes were subjected to $12 \mathrm{~h}$ of hypoxia alone $(\mathrm{H})$ or $4 \mathrm{~h}$ hypoxia plus 12 h of reoxygenation $(H / R)$. In-gel MBP kinase assays were performed. Control, Cont. Upper arrow indicates the full-length form of Mst1, while lower arrow indicates the cleaved form of Mst1. $n=3$. 
a

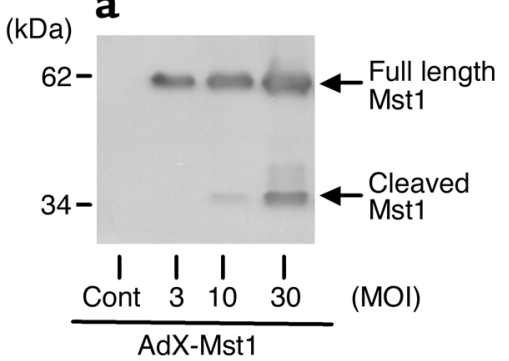

b
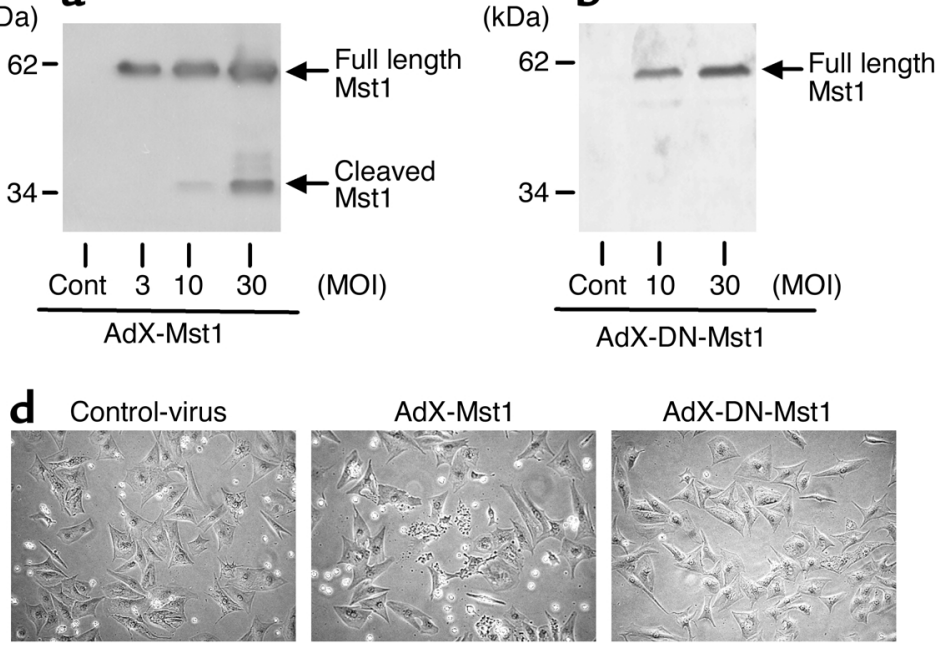

AdX-DN-Mst1

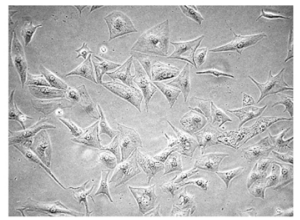

f

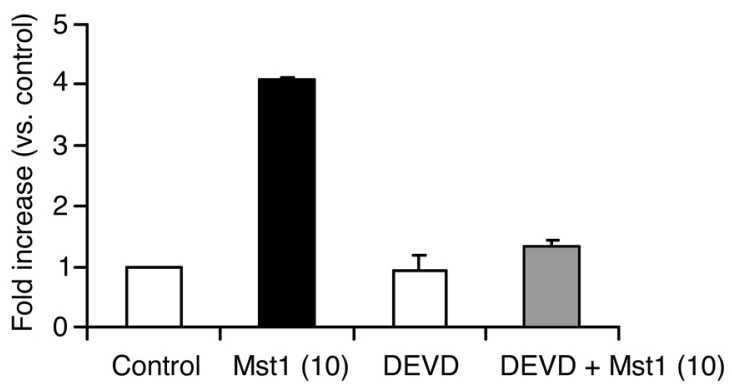

c

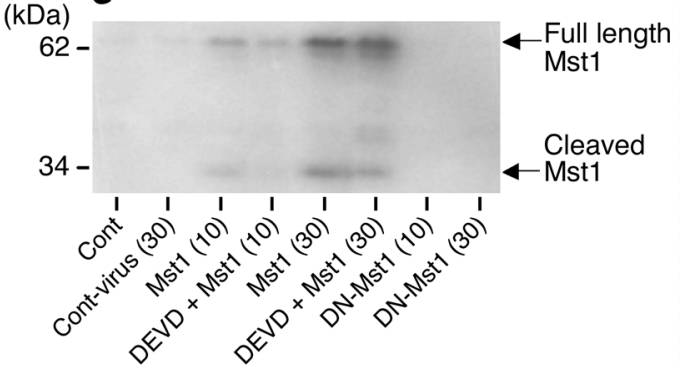

e

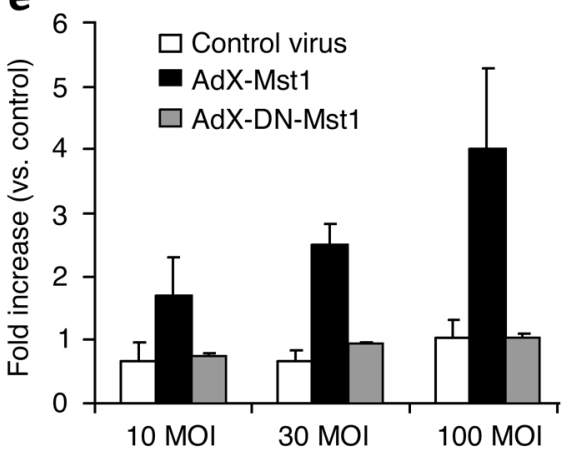

g

\section{Figure 2}

(a-g) Cardiac myocytes were transduced with adenovirus harboring either wild-type Mst1 (AdX-Mst1), AdX-DN-Mst1, or control adenovirus (Ad5 $\beta \mathrm{gal}$ ) at indicated concentrations. Myocytes were harvested $48 \mathrm{~h}$ after transduction. Some myocytes (c and $\mathbf{f}$ ) were treated with a caspase-3 inhibitor (DEVD-CHO, $100 \mu \mathrm{M}$ ). (a and b) Immunoblot analyses were performed using anti-Mst1 polyclonal Ab. Cont, control, where no virus was applied. $n=4$. (c) In-gel MBP kinase assays were performed. $n=5$. (d) The effect of adenovirus transduction (30 MOI) upon the morphology of cardiac myocytes is shown. Note that cell death with shrinkage is observed in AdX-Mst1-transduced cardiac myocytes. $n=5$. (e and $\mathbf{f}$ ) Cytoplasmic accumulation of mono- and oligo-nucleosomes, a sensitive indicator of DNA fragmentation by apoptosis, was quantitated by Cell Death ELISA Plus. (e) Myocytes were transduced with indicated doses of either control virus, AdX-Mst1, or AdX-DN-Mst1. The experimental data are normalized by those obtained in control myocytes without adenovirus transduction. $n=3$. (f) Myocytes were transduced with AdX-Mst1 (10 MOI) in the presence or absence of DEVD-CHO (100 $\mu M)$. $n=3$. (g) Activation of caspase- 3 was determined by immunoblot analyses with anti-cleaved caspase-3 Ab. $n=3$.

cross sections. The heart sections were then incubated with a $1 \%$ triphenyltetrazolium chloride solution at $37^{\circ} \mathrm{C}$ for $10 \mathrm{~min}$. The infarct area (pale), the AAR (not blue), and the total LV area from both sides of each section were measured by using Adobe Photoshop (Adobe Systems Inc., Mountain View, California, USA), and the values obtained were averaged. The weight of each section was measured using a balance. The percentage of area of infarction and AAR of each section were multiplied by the weight of the section and then totaled from all sections. AAR/LV and infarct area/AAR were expressed as a percentage. There was no significant difference in AAR/LV between Tg-DN-Mst1 and nontransgenic littermate control.

Statistics. All data are reported as mean plus or minus SEM. Statistical analyses between groups were done by one-way ANOVA, and when $F$ values were significant at a $95 \%$ confidence limit, differences among group means were evaluated using Fisher's project least significant difference post test procedure for group data with a $P$ value less than 0.05 considered significant.

\section{Results}

Mst1 is a predominant MBP kinase activated by potent stimulators of apoptosis in cardiac myocytes. To identify the signaling mechanism inducing apoptosis in cardiac myocytes, cardiac myocytes were treated with chelerythrine, a potent inducer of apoptosis (35). The wholecell extracts were subjected to in-gel kinase assays, using MBP as a substrate. We found that a $34-\mathrm{kDa}$ kinase is prominently activated by chelerythrine at 6-10 $\mu \mathrm{M}$, the concentrations where apoptosis and activation of caspase- 3 are observed (35). Activation of the 34-kDa kinase was accompanied by disappearance of a $61-\mathrm{kDa}$ kinase (Figure 1a). Longer exposures of the gel showed that chelerythrine also increased the activity of p40, p46, p54, p58, and p105, although the 


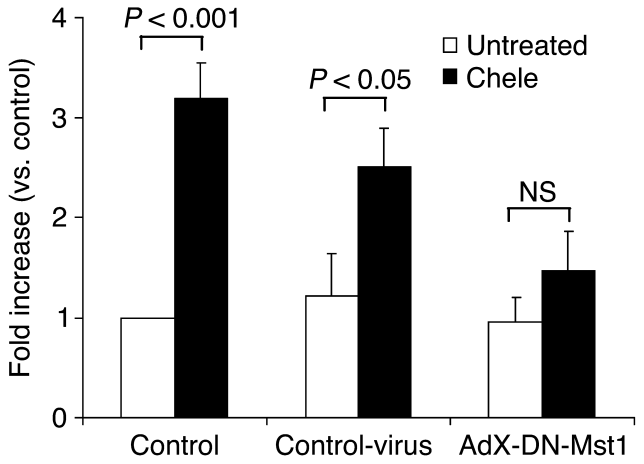

Figure 3

The effect of dominant-negative Mst1 upon chelerythrine-induced cardiac myocyte apoptosis. Cardiac myocytes were transduced with either no virus (control), control virus, or adenovirus harboring dominant-negative Mst1 (AdX-DN-Mst1). Forty-eight hours after transduction, cardiac myocytes were treated with chelerythrine $(10 \mu \mathrm{M})$ for $1 \mathrm{~h}$ and cytoplasmic accumulation of mono- and oligo-nucleosome was quantitated. $n=3$.

kinase activity of these proteins was much weaker than that of the 34-kDa kinase (not shown). Since it has been shown in other cell types that Mst1, a 61-kDa kinase, is cleaved by caspases and a resultant $34-\mathrm{kDa}$ fragment containing the $\mathrm{N}$-terminal kinase domain becomes active, we examined if the $61-\mathrm{kDa}$ and 34$\mathrm{kDa}$ proteins are Mst 1 in our experiments. Immune complex kinase assays, using specific anti-Mst1 $\mathrm{Ab}$ raised against the $\mathrm{N}$-terminal 15 -amino acids of Mst1, indicated that the $34-\mathrm{kDa}$ form of Mst 1 is activated by the chelerythrine treatment (Figure 1b). Furthermore, immunoblot analyses, using specific anti-Mst $1 \mathrm{Ab}$ 's, indicated that the $61-\mathrm{kDa}$ form of Mst 1 is downregulated, while the $34-\mathrm{kDa}$ form of Mst 1 is concomitantly upregulated after chelerythrine treatment (Figure 1c), consistent with the changes in activities of the 61- and 34$\mathrm{kDa}$ proteins in the in-gel kinase assays. To examine if Mst1 is activated by caspasedependent cleavage in cardiac myocytes, we transduced cardiac myocytes with XIAP, an endogenous inhibitor of caspases, which we have shown inhibits apoptosis in cardiac myocytes (35). Activation and cleavage of Mst 1 was completely inhibited in the presence of XIAP (Figure 1b), suggesting that Mst1 is activated by caspase-dependent cleavage in cardiac myocytes.

Recent evidence suggests that Mst 1 is also activated by phosphorylation of the activation loop even in the absence of cleavage (45). Calyculin $\mathrm{A}$, an inhibitor of protein phosphatase 2A (PP-2A) and PP-1, caused prominent cell death consistent with apoptosis in cardiac myocytes, which was evidenced by significant increases (4.3- \pm 1 .4-fold, $n=4, P<0.05$ versus untreated) in cytoplasmic accumulation of oligo- and mono-nucleosomes, sensitive indicators of nuclear fragmentation by apoptosis. In-gel kinase assays indicated that a $61-\mathrm{kDa}$ form of Mst1 is strongly activated by calyculin A without activation of the 34-kDa form (Figure 1d). Activation of Mst1 is also induced by hypoxia/reoxygenation, a known stimulus of apoptosis in cardiac myocytes (8). In this case, increases in the kinase activity were observed in both full-length and cleaved forms of Mst1 (Figure 1e). These results suggest that Mst 1 is a prominent MBP kinase, which is activated by apoptotic stimuli in cardiac myocytes. Furthermore, activation of Mst1, in both cleaved (34-kDa) and full-length (61$\mathrm{kDa}$ ) forms, is accompanied by apoptotic cell death in cardiac myocytes.

Mst1 plays an essential role in mediating apoptosis in response to chelerythrine and calyculin A in cardiac myocytes. To examine if Mst1 promotes apoptosis, we overexpressed either wild-type Mst1 or dominant-negative Mst1 (K59R) in cardiac myocytes using adenovirus transduction. Immunoblot analyses with anti-Mst $1 \mathrm{Ab}$ indicated that transduction of adenovirus harboring wild-type Mst1 (AdX-Mst1) dose dependently increased the full-length form of Mst1. Expression of the cleaved form of Mst1 was also observed at high doses, suggesting that overexpression of Mst 1 alone can induce partial cleavage of Mst1 (Figure 2a). By contrast, transduction of adenovirus harboring Mst1(K59R) (AdX-DN-Mst1) increased only the full-length form of Mst1 (K59R) (Figure 2b). a
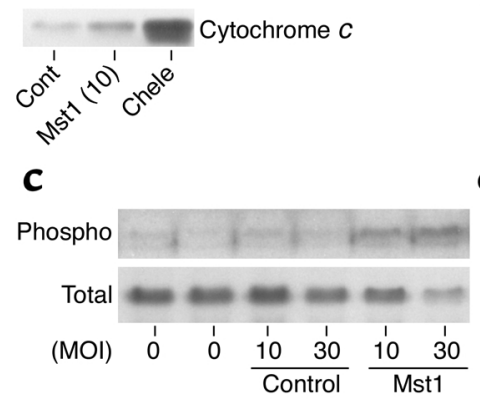

\section{Figure 4}

(a) Cardiac myocytes were transduced with either control virus (Cont) or AdXMst1 (Mst1) at $10 \mathrm{MOI}$ for $48 \mathrm{~h}$. As positive control, myocytes were treated with chelerythrine (Chele, $10 \mu \mathrm{M}$ ) for $1 \mathrm{~h}$. The mitochondria-free cytosolic fraction was obtained. Western blot analysis was performed using anti-cytochrome $c \mathrm{Ab}$ as described (35). Cytochrome $c$ oxidase IV immunoreactivity was negligible in these samples. Chele caused a release of cytochrome $c$ to the cytosolic fraction. Expression of Mst1 increased release of cytochrome $c . n=3$. (b and $\mathbf{c}$ ) Cardiac myocytes were transduced with either control virus or AdX-Mst1(Mst1) at indicated MOls for $48 \mathrm{~h}$. Immunoblot analyses were conducted using anti-phospho p38-MAPK Ab (upper) (b) or anti-phospho JNK Ab (c). In c, phosphorylation of p46-JNK is shown. The filters were reprobed with anti-p38-MAPK Ab (b) or antiJNK1 Ab (lower) (c). In b and c, Similar results were obtained in four experiments. (d) Cardiac myocytes were transduced with either control virus or AdX-DN-Mst1 virus. Myocytes were then stimulated with or without chelerythrine (Chele, 10 $\mu \mathrm{M})$ for $60 \mathrm{~min}$. Myocyte lysates were subjected to immunoblot analysis using anti-phospho p38-MAPK Ab. The filter was reprobed with anti-p38-MAPK Ab. Similar results were obtained in three experiments. 
In-gel kinase assays showed that the MBP kinase activity of Mst 1 was significantly increased by overexpression of wild-type Mst1, while it was not activated by Mst1 (K59R), confirming that Mst1 (K59R) is kinase inactive (Figure 2c). Furthermore, increased activities and cleavage of Mst 1 by overexpression of Mst1 were attenuated in the presence of DEVD-CHO, an inhibitor of caspase- 3 and its related subfamily, suggesting that Mst 1 activates caspases, which in turn causes cleavage of Mst1 (Figure 2c, lane 4). Transduction of either control virus or AdX-DN-Mst1 did not induce any significant changes in the morphology of cardiac myocytes. By contrast, transduction of AdX-Mst 1 caused shrinkage and cell death in cardiac myocytes (Figure 2d). Cytoplasmic accumulation of mono- and oligo-nucleosomes was dose dependently increased by overexpression of wild-type Mst1, but not by control virus or Mst1 (K59R) (Figure 2e). Increases in DNA fragmentation by wild-type Mst1 were inhibited in the presence of DEVD-CHO (Figure 2f). Overexpression of wild-type Mst1, but not Mst1 (K59R), caused activation of caspase-3, which was determined by immunoblotting with anti-cleaved specific caspase- $3 \mathrm{Ab}$ (Figure $2 \mathrm{~g}$ ). These results suggest that Mst1 induces cardiac myocyte apoptosis in a kinase activity-dependent manner.

To examine if Mst 1 is required for cardiac myocyte apoptosis in response to chelerythrine, cardiac myocytes were transduced with AdX-DN-Mst 1 or control virus and challenged by chelerythrine. Cytoplasmic accumulation of mono- and oligo-nucleosomes by chelerythrine treatment was significantly suppressed in the presence of AdX-DN-Mst1, but not by control virus (Figure 3). DNA fragmentation by calyculin A was also inhibited by AdX-DN-Mst1 (5.6-fold with control virus versus 0.9 -fold with AdX-DN-Mst1). These results suggest that both cleaved and fulllength forms of Mst 1 play a critical role in mediating apoptosis in cardiac myocytes.

We further examined the mechanism by which Mst1 stimulates apoptosis in cardiac myocytes. Transduction of AdX-Mst1 significantly (twofold, $n=3, P<0.05$ ) increased the amount of cytochrome $c$ in the mitochondria-free cytosolic fraction compared with that of control virus, suggesting that release of cytochrome $c$ may contribute to the proapoptotic effect of Mst 1 (Figure 4a). Transduction of AdX-Mst 1 modestly activated p38-MAPK and p46-JNKs (Figure 4, b and c), while that of AdX-DN-Mst 1 abolished chelerythrine-induced activation of p38-MAPK in cardiac myocytes (Figure 4d). These results suggest that Mst1 works as MAP4K in cardiac myocytes and that it plays an important role in mediating activation of p38-MAPK by chelerythrine.

Cardiac-specific overexpression of Mst 1 in mice causes dilated cardiomyopathy with increases in TUNEL-positive cells and activation of caspase-3. To examine the function of Mst 1 in the mouse heart in vivo, transgenic mice with cardiac-specific overexpression of wild-type Mst1 (Tg-Mst1) were generated using the $\alpha \mathrm{MHC}$ promoter. We identified three

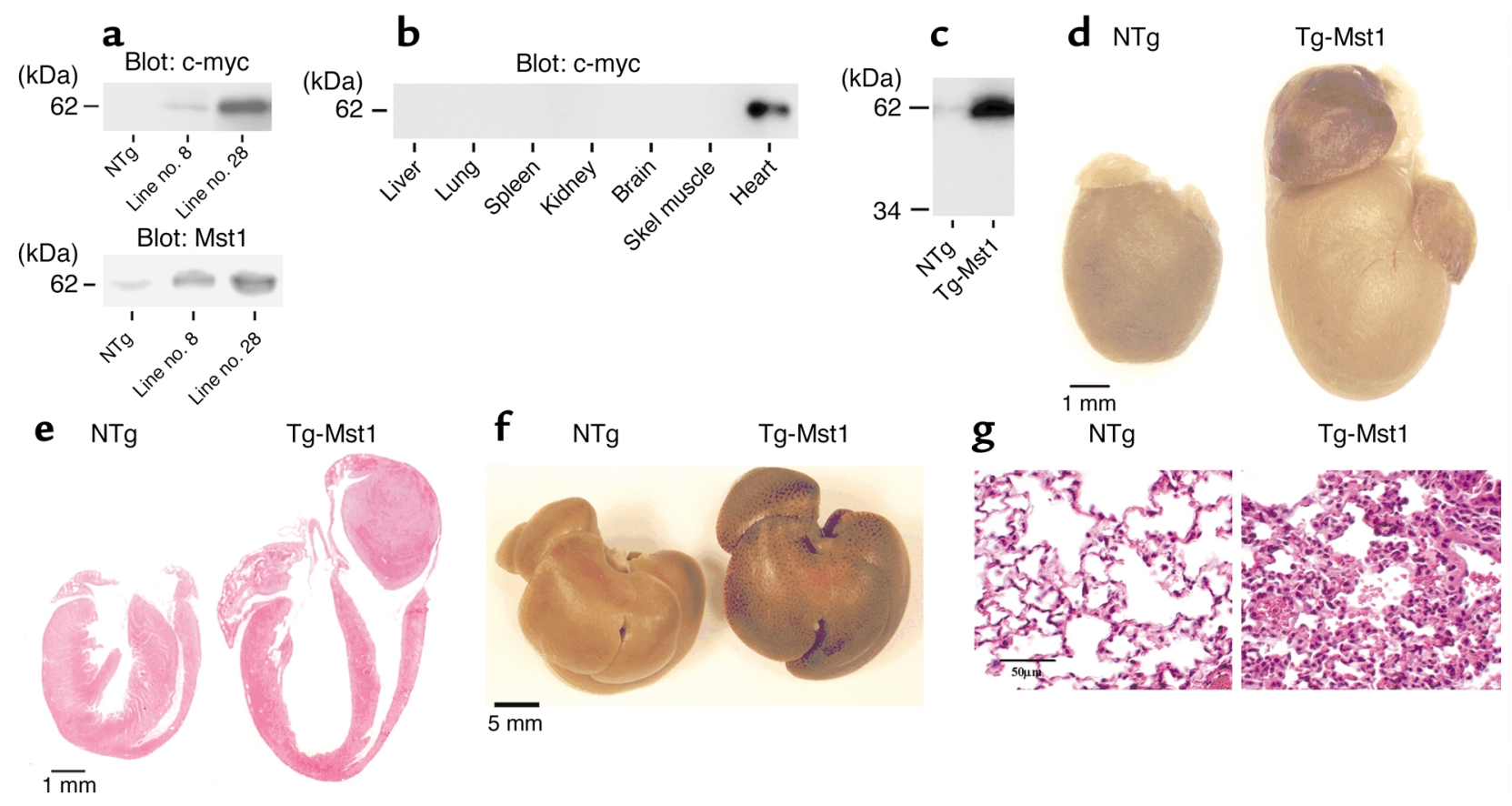

Figure 5

(a) Immunoblot analyses of heart homogenates with anti-myc Ab (upper panel). Note that Myc-Mst1 migrates at $62 \mathrm{kDa}$. Immunoblots were also conducted using anti-human Mst1 Ab (lower panel). (b) Tissue homogenates were prepared from various organs of Tg-Mst1. Immunoblot analyses were performed with anti-myc Ab. (c) Heart homogenates were prepared from Tg-Mst 1 or nontransgenic control mice $(\mathrm{NTg})$. In-gel MBP kinase assays were performed. $n=3$. ( $\mathbf{d}$ and $\mathbf{e})$ Gross appearance and a transverse section of the hearts obtained from Tg-Mst 1 and NTg. In e, hematoxylin-eosin staining was performed (96 days). (f) A photograph of the liver isolated from Tg-Mst1 and littermate NTg (96 days). (g) Hematoxylin-eosin staining of the lung. 
Table 1

Echocardiographic analyses of Tg-Mst1

\begin{tabular}{lcccc} 
& \multicolumn{2}{c}{ Line 28 (high-level overexpression) } & \multicolumn{2}{c}{ Line 8 (intermediate level overexpression) } \\
& Nontransgenic $(n=4)$ & TG-Mst1 $(n=5)$ & Nontransgenic $(n=4)$ & TG-Mst1 $(n=5)$ \\
Age $($ days old) & $75 \pm 2.89$ & $76 \pm 2.45$ & $195 \pm 1.96$ & $193 \pm 1.96$ \\
LVEDD $(\mathrm{mm})$ & $3.92 \pm 0.11$ & $4.39 \pm 0.06^{\mathrm{A}}$ & $4.40 \pm 0.25$ & $4.54 \pm 0.28$ \\
LVESD $(\mathrm{mm})$ & $2.62 \pm 0.11$ & $3.55 \pm 0.10^{\mathrm{A}}$ & $2.99 \pm 0.14$ & $3.29 \pm 0.19$ \\
LVEF $(\%)$ & $70.0 \pm 1.83$ & $47.2 \pm 2.80^{\mathrm{A}}$ & $68.4 \pm 1.21$ & $61.6 \pm 2.38^{\mathrm{B}}$ \\
\%FS (\%) & $33.1 \pm 1.44$ & $19.2 \pm 1.41^{\mathrm{A}}$ & $31.9 \pm 0.78$ & $27.5 \pm 1.46^{\mathrm{B}}$ \\
DSEP WT $(\mathrm{mm})$ & $0.59 \pm 0.04$ & $0.39 \pm 0.01^{\mathrm{A}}$ & $0.68 \pm 0.02$ & $0.64 \pm 0.03$ \\
DPW WT $(\mathrm{mm})$ & $0.59 \pm 0.02$ & $0.37 \pm 0.03^{\mathrm{A}}$ & $0.65 \pm 0.02$ & $0.63 \pm 0.02$ \\
HR (bpm) & $246.8 \pm 23.4$ & $303.0 \pm 32.8$ & $258.2 \pm 8.1$ & $255.8 \pm 25.2$ \\
\hline
\end{tabular}

${ }^{A} P<0.01$ compared with nontransgenic; ${ }^{B} P<0.05$ compared with nontransgenic. DSEPWT, diastolic septal wall-thickness; DPW WT, diastolic posterior wall wall-thickness.

transgene positive founders by Southern blot analyses. Among them, germline transmission was observed in two lines. One line (no. 28) showed prominent cardiac specific overexpression of wild-type Mst1, while the other line (no. 8) showed modest overexpression (Figure 5a). In both lines, approximately $50 \%$ of offspring was shown to carry the transgene. In this study the line no. 28 was predominantly characterized, while some analyses were conducted using line no. 8, as well. We confirmed that Mst1 is overexpressed predominantly in the heart (Figure $5 b$ ). In-gel MBP kinase assays indicated that the total activity of Mst 1 was significantly increased (7.5-fold) in Tg-Mst1. Increases in the MBP kinase activity in Tg-Mst1 were found predominantly in the full-length form rather than in the cleaved form (Figure 5c).

Interestingly, some Tg-Mst 1 exhibited overt signs of heart failure, such as shortness of breath and edema and died prematurely as early as on day 15 . Echocardiographic measurements of Tg-Mst1(line no. 28) at 70-80 days old indicated that Tg-Mst 1 exhibited significant increases in LV end-diastolic dimension (LVEDD) and LV end-systolic dimension (LVESD) and significant decreases in LV ejection fraction (LVEF), LV fractional shortening (\%FS) and LV wall thickness (Table 1). Smaller but significant decreases in LVEF and \%FS were also noted in line no. 8 of Tg-Mst 1 at the age of 6-7 months (Table 1). Hemodynamic analyses confirmed that LVEDP was significantly elevated, while LV first derivative of pressure $(\mathrm{dP} / \mathrm{dt})$ was decreased in Tg-Mst 1 compared with nontransgenic littermates (Table 2). Necropsy of Tg-Mst 1 at 3 months old indicated dilation of all four cardiac chambers, mural thrombus formation in both atriums, and reduced wall thickness, consistent with the findings of dilated cardiomyopathy (Figure 5, d and e). LV weight/body weight and RV weight/body weight were not significantly different between Tg-Mst 1 and nontransgenic littermates at all ages (Table 3 and data not shown). Significant increases in lung weight/ body weight and liver weight/body weight were observed in Tg-Mst 1 compared with nontransgenic littermates at 1.5 months old (Table 3). In later stages (at 3-4 months old), visible congestion of the liver and the lungs was observed in Tg-Mst1 (Figures 5, f and g).
To evaluate the frequency of cell death in the myocardium, TUNEL staining was performed on 1- to 2-month-old mice. TUNEL-positive myocytes are significantly increased in Tg-Mst1 (line 28). Smaller but significant increases in TUNEL-positive myocytes were also observed in the line 8 of Tg-Mst1 (Figure 6a). The level of cleaved caspase- 3 was increased in hearts of Tg-Mst 1 (Figure 6b), suggesting that caspase- 3 is activated. These results suggest that cardiac myocyte death consistent with apoptosis is enhanced in hearts of $\mathrm{Tg}$-Mst1. It should be noted that the occurrence of oncotic cell death cannot be excluded in $\mathrm{LV}$ myocardium of TgMst1, since some myocytes exhibited formation of vacuoles consistent with myolysis (Figure $6 c$, indicated by an arrow). The level of interstitial fibrosis was significantly increased in all four chambers of Tg-Mst1 (Figure 6d), consistent with the notion that myocyte death and subsequent replacement of myocardium with fibrous tissue take place in Tg-Mst1 hearts. Infiltration of inflammatory cells was generally not significant in hearts of Tg-Mst1 (Figure 6e), although mild infiltration was very occasionally found at a site of myolysis (Figure 6c). We further tested if increased cell death causes decreases in the myocyte density of the myocardium in Tg-Mst 1 . The myocyte density in the LV myocardium was significantly reduced in Tg-Mst1 (Figure 6f).

\section{Table 2}

Hemodynamic measurements of Tg-Mst1

\begin{tabular}{lcc}
\hline & Nontransgenic $(n=4)$ & Tg-Mst $1(n=5)$ \\
Age (days) & $80 \pm 7.8$ & $88 \pm 12$ \\
LVSP $(\mathrm{mm} \mathrm{Hg})$ & $84 \pm 2.3$ & $70 \pm 5.1$ \\
LVEDP $(\mathrm{mm} \mathrm{Hg})$ & $3.3 \pm 2.9$ & $18.6 \pm 1.5^{\mathrm{B}}$ \\
$+\mathrm{LV} \mathrm{dP} / \mathrm{dt}$ & $5,200 \pm 245$ & $4,300 \pm 663$ \\
$-\mathrm{LV} \mathrm{dP} / \mathrm{dt}$ & $4,467 \pm 356$ & $3,080 \pm 481$ \\
$\mathrm{RVSP}(\mathrm{mm} \mathrm{Hg})$ & $18 \pm 2.9$ & $29 \pm 3.3^{\mathrm{A}}$ \\
$\mathrm{RVEDP}(\mathrm{mm} \mathrm{Hg})$ & $0.7 \pm 0.4$ & $9.0 \pm 2.2^{\mathrm{A}}$ \\
$\operatorname{RAP}(\mathrm{mm} \mathrm{Hg})$ & $1.3 \pm 0.4$ & $7.0 \pm 2.3$ \\
HR $(\mathrm{bpm})$ & $323 \pm 54$ & $297 \pm 36$
\end{tabular}

${ }^{A} P<0.05 ;{ }^{B} P<0.001$ compared with nontransgenic. LVSP, left ventricular systolic pressure; LVEDP, left ventricular end-diastolic pressure; RVEDP, right ventricular end-diastolic pressure; RAP, right atrial pressure. 
Table 3

Postmortem pathologic measurements of Tg-Mst1

\begin{tabular}{lcc}
\hline & Nontransgenic $(n=9)$ & Tg-Mst1 $(n=12)$ \\
Age (days old) & $46.7 \pm 2.7$ & $46.9 \pm 1.9$ \\
Body weight $(\mathrm{g})$ & $18.4 \pm 0.4$ & $19.4 \pm 0.8$ \\
LV $(\mathrm{mg})$ & $64.2 \pm 2.0$ & $64.5 \pm 2.6$ \\
RV $(\mathrm{mg})$ & $19.1 \pm 0.8$ & $20.6 \pm 0.6$ \\
LV/body weight & $3.48 \pm 0.07$ & $3.32 \pm 0.06$ \\
Lung/body weight & $6.67 \pm 0.30$ & $7.73 \pm 0.29^{\mathrm{A}}$ \\
Liver/body weight & $47.6 \pm 1.78$ & $55.1 \pm 2.71^{\mathrm{A}}$ \\
\hline
\end{tabular}

${ }^{A} P<0.05$ compared with nontransgenic.

Compensatory cardiac myocyte hypertrophy is absent in $T g-M s t 1$. Increased LV diameter and reduced wall thickness at a given pressure increases LV wall stress in Tg-Mst 1 . To examine if there is compensatory hypertrophy at the level of cardiac myocytes, the myocyte size was determined by three independent methods. The cross-sectional area of $\mathrm{LV}$ cardiac myocytes, determined by silver staining, was not significantly different between Tg-Mst 1 and nontransgenic mice (Figure 7, a and $b$ ). The longitudinal length of ventricular myocytes isolated from Tg-Mst1 was actually significantly smaller than those from nontransgenic controls (Figure 7c). Furthermore, the cellular capacitance, which reflects the cell volume, was significantly smaller in ventricular myocytes from Tg-Mst 1 than in those from nontransgenic mice (Figure 7d). These results indicate that there was no compensatory cardiac myocyte hypertrophy in Tg-Mst1. To test if side-to-side slippage of myocytes takes place in the ventricular wall of Tg-Mst 1 , the number of myocyte across the LV free wall was counted. The mural number of myocytes was significantly less in Tg-Mst 1 than in nontransgenic mice (Figure 7e), suggesting that lateral myocyte slippage may be the mechanism mediating cardiac dilation in Tg-Mst 1 .

Cardiac-specific overexpression of Mst1 (K59R) works as dominant-negative Mst1 and significantly reduces apoptosis in response to $I / R$. To examine if activation of endogenous Mst 1 is required for apoptosis in response to pathologic insults to the heart, we generated transgenic mice with cardiac-specific overexpression of dominant-negative Tg-DN-Mst1. Among three lines generated, line 10 expressed the highest level of Tg-DN-Mst 1 in the heart (Figure 8a), while expression of transgene in other lines (lines 9 and 11) was very low. Tg-DN-Mst 1 did not show premature death or any signs of heart failure. The results of echocardiographic measurements of Tg-DN-Mst 1 were not significantly different from those of nontransgenic littermates (data not shown). These results suggest that development of dilated cardiomyopathy in Tg-Mst 1 is dependent upon the kinase activity of Mst 1 . To confirm that overexpression of Mst1(K59R) works as a dominant-negative, we applied $20 \mathrm{~min}$ of ischemia and subsequent $24 \mathrm{~h}$ of reperfusion to the mouse heart. Results of in-gel MBP kinase assays showed that $\mathrm{I} / \mathrm{R}$ activates Mst1 primarily in the full-length
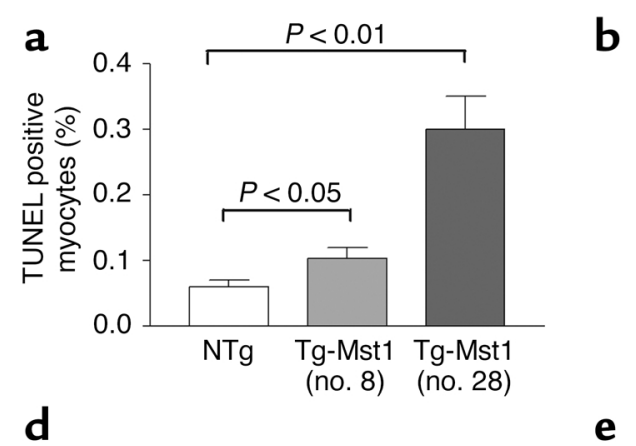

d

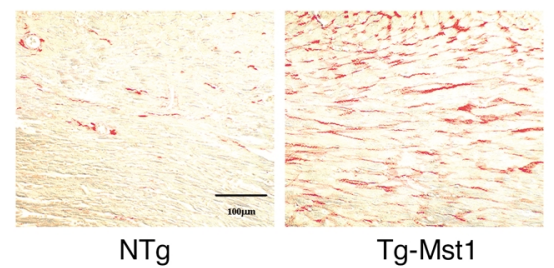

b

e
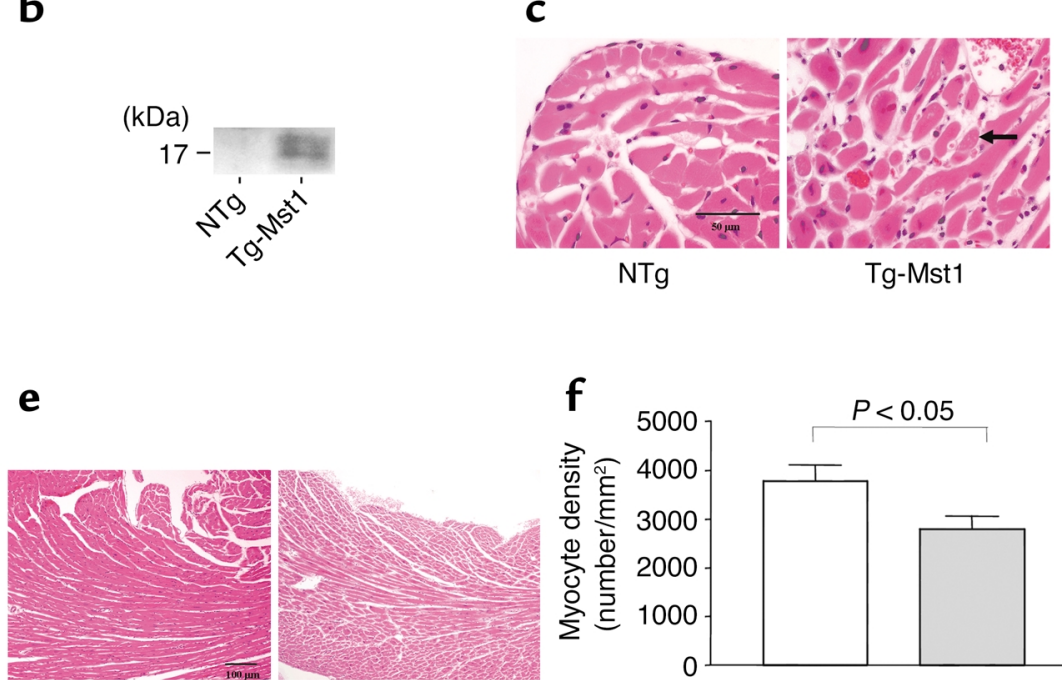

$\mathrm{NTg}$

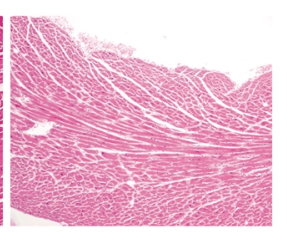

Tg-Mst1

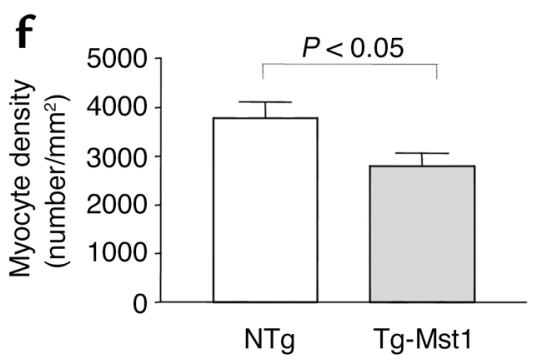

Figure 6

(a) TUNEL-positive myocytes in the LV myocardium. We analyzed five Tg-Mst1 (no. 8), four Tg-Mst1 (no. 28 ), and nine control nontransgenic littermates (NTg). The number of TUNEL-positive myocytes was expressed as percentage of total nuclei determined by DAPI staining. (b) Heart homogenates were prepared from Tg-Mst1 and NTg. Immunoblot analyses were performed using anti-cleaved caspase-3 Ab. $n=3$. (c) Hemotoxylin-eosin staining of the myocardium. Myocytes with vacuoles (shown by an arrow) are very occasionally found in the LV myocardium of Tg-Mst1. In this area, mild increases in infiltration of inflammatory cells are found. (d) Picric acid Sirius red staining of heart sections obtained from Tg-Mst1 and NTg (96 days old). (e) Hematoxylin-eosin staining of the LV myocardium. Note that infiltration of inflammatory cells are generally not significant in Tg-Mst1. (f) The number of myocytes per 1 mm ${ }^{2}$ was determined histologically from the sections obtained from LV myocardium. We studied five NTg and seven Tg-Mst1. 
form in control mouse hearts (Figure 8b). By contrast, activation of Mst 1 in response to $\mathrm{I} / \mathrm{R}$ was abolished in Tg-DN-Mst1, suggesting that cardiac-specific overexpression of Mst1 (K59R) works as a dominant-negative for endogenous Mst1 (Figure 8b). The heart section obtained from the ischemic area of Tg-DN-Mst1 exhibited a significantly smaller number of TUNELpositive myocytes compared with that of nontransgenic littermates (Figures 8, $\mathrm{c}$ and d). Electrophoresis of genomic DNA prepared from the ischemic area of nontransgenic mice exhibited a typical pattern of DNA laddering. By contrast, DNA laddering was significantly suppressed in Tg-DN-Mst1 (Figure 8e). The extent of myocardial infarction in the area at risk was significantly smaller in Tg-DN-Mst 1 compared with that in nontransgenic littermates (Figure 8f). These results suggest that inhibition of Mst1 reduces both cardiac myocyte apoptosis and the size of myocardial infarction in response to $\mathrm{I} / \mathrm{R}$ in mouse hearts.

\section{Discussion}

Our results suggest that Mst 1 is activated by clinically important pathologic insults such as $\mathrm{I} / \mathrm{R}$ and that activation of Mst 1 is necessary and sufficient in mediating cardiac myocyte apoptosis by such stresses. Cardiac-specific overexpression of Mst 1 in mice resulted in a dilated cardiomyopathy. Unlike other dilated cardiomyopathies, the size and length of individual myocytes were reduced, thereby possibly making operation of the Frank-Starling mechanism less efficient at the single cell level. This represents a novel mechanism of congestive heart failure and suggests that
Mst 1 could be an important target of treatment for congestive heart failure.

Mst1 is a predominant MBP kinase activated by potent proapoptotic stimuli in cardiac myocytes. Our results suggest that Mst 1 is the most prominent MBP kinase activated by potent apoptotic stimuli, including chelerythrine and calyculin A, in cardiac myocytes. Activation of Mst1 was also observed in response to hypoxia/reoxygenation of cardiac myocytes in vitro and $\mathrm{I} / \mathrm{R}$ of the mouse heart in vivo. Activation of Mst 1 by such clinically relevant stresses has not been reported previously. Overexpression of wild-type Mst1 was sufficient to cause typical findings of apoptosis in cardiac myocytes. Since cleavage and activation of Mst1 were in part inhibited by the caspase inhibitor, activation of Mst 1 partially depends upon caspases. Thus, Mst1 and caspases stimulate one another, thereby initiating a positive feedback mechanism leading to increased apoptosis in cardiac myocytes. These results suggest that Mst 1 can be an important regulator of apoptosis in cardiac myocytes.

Recent evidence suggests that full-length and cleaved forms of Mst 1 are activated by phosphorylation of the catalytic domain and by caspase-mediated cleavage of the C-terminal inhibitory domain, respectively (45). In fact, Mst1 is activated by both mechanisms in cardiac myocytes: chelerythrine causes activation of Mst1 via caspase-dependent cleavage, while calyculin A causes activation of the full-length form of Mst1, possibly through phosphorylation. Mst1 may be activated through both mechanisms in response to hypoxia/reoxygenation. Since cardiac a

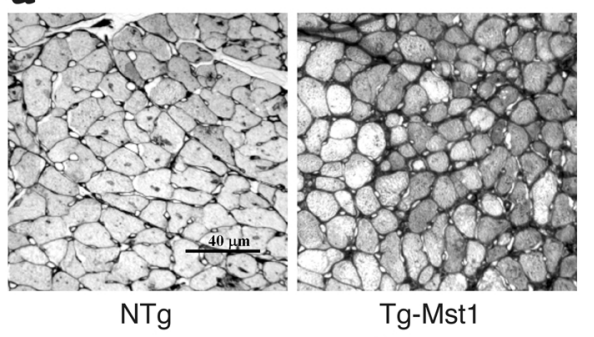

d

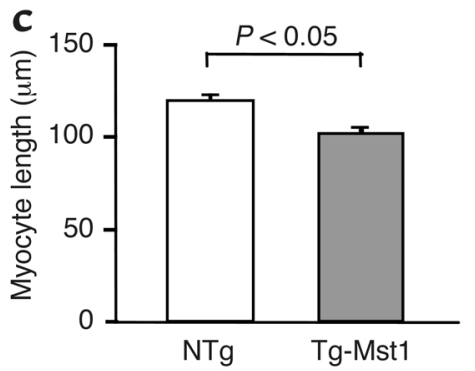

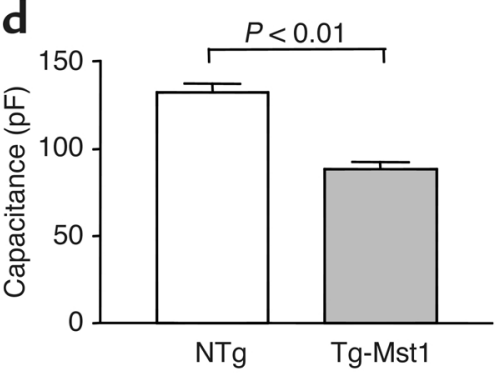

b
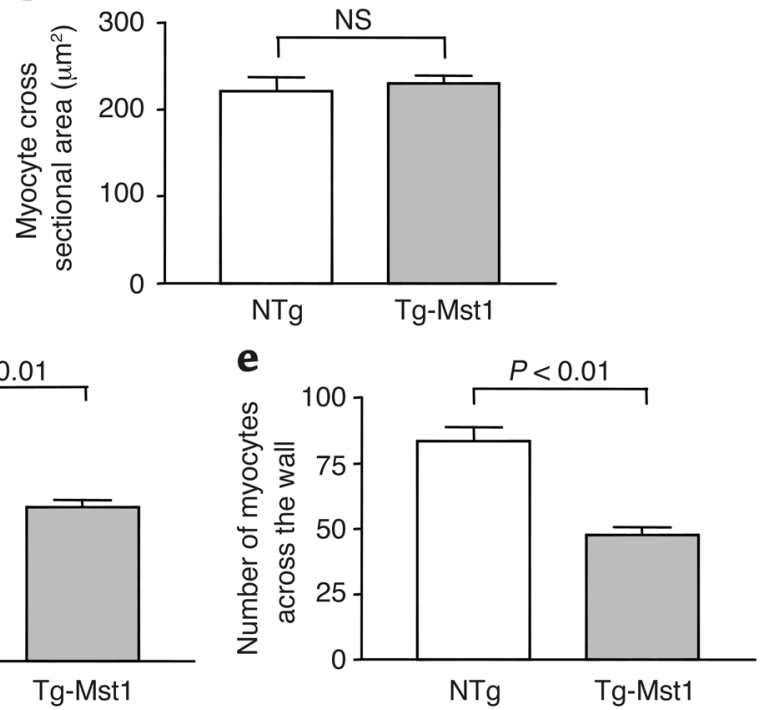

\section{Figure 7}

(a and b) LV cardiac myocyte cross sectional area was obtained from Tg-Mst1 and nontransgenic control mice (NTg) as described in Methods. Representative silver staining of the LV myocardium obtained from NTg and Tg-Mst1 are shown in a. Seven Tg-Mst1 and four NTg mice were used for the analysis in b. (c) The longitudinal myocyte length was significantly reduced in LV myocytes from Tg-Mst 1 ( $n=4,111$ cells) compared with NTg $(n=4,129$ cells). (d) Average cell capacitance of LV cardiac myocytes obtained from NTg and Tg-Mst1 ( $n=21)$. (e) The number of cardiac myocyte across the LV free wall was significantly reduced in Tg-Mst1 compared with that in NTg. We studied five NTg and seven Tg-Mst1. 
a<smiles>CC(C)C(=O)Oc1ccccc1</smiles>

(kDa) Blot: c-Mst1

62<smiles>[CH]1[C@H]2C[C@H]1C2</smiles><smiles>c1ccccc1</smiles>

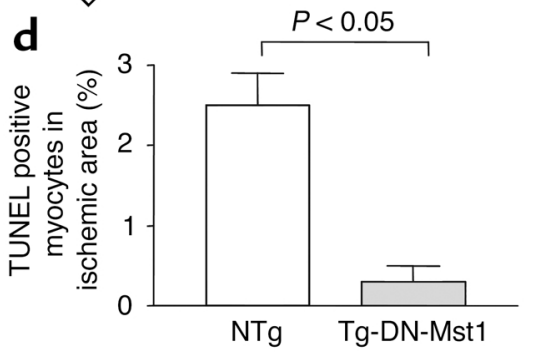

C

(kDa)
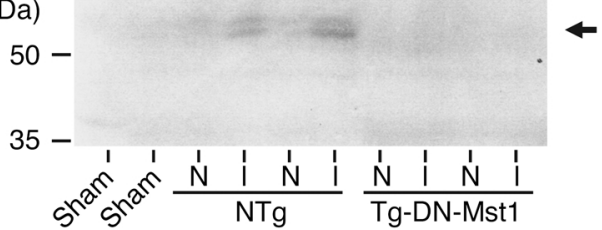

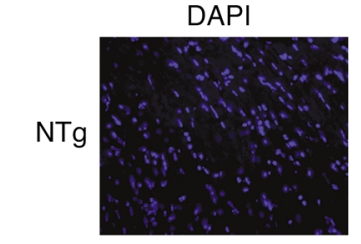

Tg-DN-Mst1

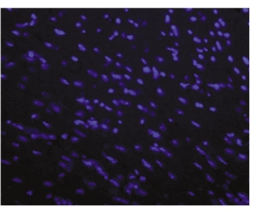

e

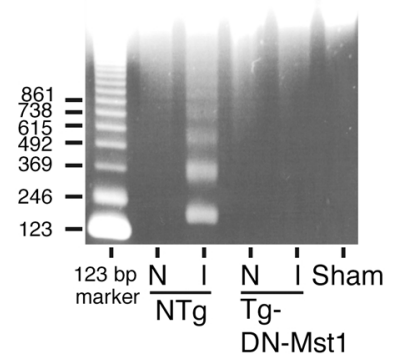

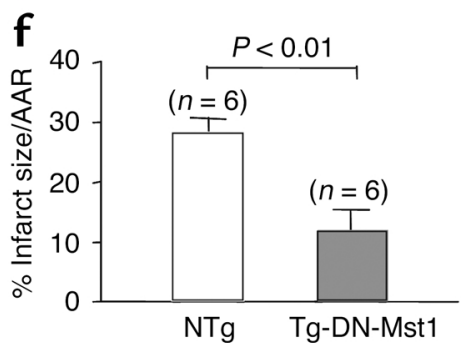

Figure 8

(a) Immunoblot analyses of heart homogenates with anti-myc Ab (upper panel) and anti-Mst1 Ab (lower panel). (b-f) Tg-DN-Mst1 or NTg control mice were subjected to $20 \mathrm{~min}$ ischemia and $24 \mathrm{~h}$ of reperfusion or sham operation. (b) The heart homogenates (100 $\mu \mathrm{g})$ obtained from ischemic $(\mathrm{I})$ and nonischemic $(\mathrm{N})$ areas of the $\mathrm{LV}$ of the mice received $\mathrm{I} / \mathrm{R}$, or from intact $\mathrm{LV}$ of the sham-operated mice were subjected to in-gel MBP kinase assays. I/R increased kinase activities of full-length Mst1 in the ischemic area of NTg mice, while activation of Mst 1 by $\mathrm{I} / \mathrm{R}$ was completely abolished in Tg-DN-Mst1. A faint band seen just above $61 \mathrm{kDa}$ Mst1 most likely represents Mst2, a homologue of Mst1, whose activities are also abolished in the presence of DN-Mst1. $n=3$. (c) LV tissue sections were subjected to TUNEL staining and DAPI staining. (d) TUNEL-positive myocytes in the ischemic area. The number of TUNEL-positive myocytes was expressed as percentage of total nuclei determined by DAPI staining. $n=5$. (e) Genomic DNA was isolated from nonischemic $(\mathrm{N})$ and ischemic $(\mathrm{I})$ areas, and DNA-laddering assays were performed. The extent of DNA laddering in response to $\mathrm{I} / \mathrm{R}$ was significantly smaller in Tg-DN-Mst 1 compared with that in NTg. $n=3$. (f) The effect of I/R upon the extent of LV myocardial infarction in Tg-DN-Mst1 and NTg. The myocardial infarction area/AAR (\% infarct size/AAR) was determined as described in Methods. Note that \% infarct size/AAR was significantly smaller in Tg-DN-Mst1 than in NTg.

myocyte death by both mechanisms was inhibited by dominant-negative Mst1, activation of Mst1 by either mechanism mediates cardiac myocyte apoptosis. Interestingly, the active form of Mst 1 in Tg-Mst 1 or in control mice subjected to I/R predominantly exists in the full-length form. This indicates that cleavage of Mst 1 by previously activated caspases may not be required for activation of Mst 1 in pathological conditions in vivo. Since partial cleavage of Mst1 was observed when higher doses of AdX-Mst1 were transduced in cardiac myocytes in vitro or $\mathrm{I} / \mathrm{R}$ was applied to Tg-Mst1 (data not shown), we speculate that higher levels of caspase activation is required for the cleavage of Mst 1 to be seen. Since we could detect cleavage of caspase-3 even without cleavage of Mst 1 in Tg-Mst1, caspases may have a higher affinity for self-cleavage compared with cleavage of Mst1. Alternatively, activation of caspase- 3 and cleavage of Mst 1 could coexist when cells are undergoing apoptosis, but anticleaved caspase-3 Ab may be more sensitive for detection of the cleaved product.

The pattern of Mst 1 activation by overexpression or by $\mathrm{I} / \mathrm{R}$ is analogous to that by calyculin $\mathrm{A}$, a phosphatase inhibitor. In this regard, it will be interesting to examine if the activation loop of Mst 1 is phosphorylated in $\mathrm{Tg}-\mathrm{Mst} 1$ or in response to I/R (45). It has been reported that transgenic mice overexpressing an inhibitor of PP-2A exhibited dilated cardiomyopathy (46). It would be interesting to examine if the activation of Mst 1 is involved in development of heart failure in these animals.

Cardiac-specific overexpression of Mst1 stimulates cardiac myocyte apoptosis without compensatory cardiac myocyte bypertrophy and induces dilated cardiomyopathy. Cardiacspecific overexpression of wild-type Mst 1 dose dependently caused dilation in all four chambers, wall thinning, and reduced bi-ventricular function, consistent with findings of dilated cardiomyopathy in mice.

What is the cause of dilated cardiomyopathy in Tg-Mst1? We found that Tg-Mst1 have an increased number of TUNEL positive cells, which was accompanied by increased levels of caspase- 3 activity. Although whether the TUNEL-positive cells in Tg-Mst1 represent apoptosis or oncosis remains to be elucidated, since Mst 1 promotes cell death consistent with apoptosis in vitro, it is likely that Tg-Mst 1 mice have increased levels of apoptosis. Considering the fact that increases in TUNEL-positive myocytes were observed 
even before the animals manifest overt signs of heart failure, increases in cardiac myocyte death may contribute at least in part to initial development of dilated cardiomyopathy in Tg-Mst1. Consistent with this notion, we found that the density of cardiac myocytes in the LV myocardium is reduced in Tg-Mst1. Since the prevalence of apoptosis in Tg-Mst $1(0.3 \%)$ is similar to that in human heart failure (47), Tg-Mst1 should serve as a useful model to study the role of apoptosis in progression of congestive heart failure.

Our results suggest that Mst 1 is a MAP4K and regulates downstream SRPKs, such as $\mathrm{p} 38$-MAPK and $\mathrm{p} 46-$ JNKs in cardiac myocytes. Whether or not p38-MAPK and/or p46-JNKs mediate proapoptotic effects of Mst1 is of great interest. The activated form of Mst1 is translocated to the nucleus and may affect death-associated protein 4 (48) and the nuclear components of the apoptosis machinery, including inhibitor of caspase-activated DNase $(32,33,45)$. Although Mst1 efficiently phosphorylates MBP at least in vitro, pathophysiologically relevant substrates of Mst 1 , mediating the proapoptotic effect of Mst1, remain to be elucidated in cardiac myocytes.

Cardiac-specific overexpression of Mst 1 prevents compensatory cardiac myocyte bypertrophy and potentially obscures operation of the Frank-Starling mechanism in individual cardiac myocytes. One of the most surprising findings in this study was that hypertrophy of surviving ventricular cardiac myocytes was not observed in Tg-Mst1. To our knowledge, Mst 1 is unique among protein kinases thus far overexpressed in the mouse heart in vivo, because overexpression of Mst 1 in the heart primarily stimulates cardiac myocyte death without compensatory hypertrophy. Absence of cardiac hypertrophy not only leaves the wall stress elevated but also fails to compensate for the loss of cardiac mass caused by apoptosis in Tg-Mst1. Importantly, the longitudinal length as well as the cell volume of isolated LV myocytes were significantly smaller in Tg-Mst1. Although increases in myocyte length are commonly observed in dilated cardiomyopathy, to our knowledge, decreases in cell volume or cardiac myocyte length in dilated cardiomyopathy have not been reported previously. We speculate that side-to-side slippage of cardiac myocytes (42), rather than elongation of individual myocytes, supports ventricular dilation in Tg-Mst1. Thus, increased tension development in dilated hearts according to the Frank-Starling mechanism would become less efficient at a single-cell level in Tg-Mst1, which may further contribute to decreased cardiac function in these animals.

At present, we do not know why compensatory cardiac myocyte hypertrophy does not take place in Tg-Mst 1 despite elevated wall stress. Mst 1 may inhibit signaling molecules causing hypertrophy through either direct phosphorylation or caspase-mediated cleavage. Little is known about the cellular actions of Mst1 besides apoptosis. If Mst1 possess such direct antihypertrophic function, this would be a novel function of Mst1. Alternatively, ongoing cell proliferation could make cardiac myocytes smaller (18). In this regard, whether or not increased cell death stimulates myocyte proliferation in Tg-Mst 1 remains to be elucidated.

Mst1 plays an important role in mediating cardiac myocyte death in response to $I / R$ in the heart. Although endogenous Mst1 is activated by proapoptotic stimuli in both neonatal rat cardiac myocytes and the adult mouse heart, one may argue that overexpression of Mst 1 causes nonphysiological responses. To address this issue, we tested the effect of dominant-negative Mst1 (K59R) and confirmed that it was able to suppress the activity of endogenous Mst 1 in baseline and in response to I/R. Most importantly, cardiac-specific expression of dominant-negative Mst 1 significantly reduced the extent of myocardial infarction. Since increases in both TUNELpositive cells and DNA laddering by $I / R$ were significantly suppressed in Tg-DN-Mst1, inhibition of endogenous Mst 1 most likely reduced cardiac myocyte apoptosis. We cannot completely exclude the possibility that overexpression of Mst1 (K59R) may affect activities of other protein kinases. Since Mst 1 is a predominant MBP kinase activated by $\mathrm{I} / \mathrm{R}$, however, the effect of the Mst1 (K59R) is most likely mediated through inhibition of Mst 1 or that of the closely related Mst1 family, such as Mst2.

It has been shown recently that NORE, a noncatalytic polypeptide homologous to the putative tumor suppressor RASSF1, associates with Mst1, and the NOREMst1 complex mediates Ras-dependent apoptosis in fibroblasts (49). Thus, Mst1 may be involved in apoptosis caused by a wide variety of extracellular stimuli as well as environmental stresses. In this regard, Mst1 could be an important therapeutic target in many cardiovascular diseases.

\section{Acknowledgments}

We thank Jeffrey Robbins for providing us with the $\alpha \mathrm{MHC}$ promoter construct. This work was in part supported by grants from the NIH (HL-59139, HL-33107, HL-33065, HL-65182, HL-65183, AG-14121, HL-69020, HL-67724, HL-67727), American Heart Association National grant (9950673N), and a Postdoctoral Fellowship, American Heart Association Heritage Affiliate (0120290T).

\footnotetext{
1. Kajstura, J., et al. 1996. Apoptotic and necrotic myocyte cell death are independent contributing variables of infarct size in rats. Lab. Invest. 74:86-107.

2. Saraste, A., et al. 1997. Apoptosis in human acute myocardial infarction. Circulation. 95:320-323.

3. Tanaka, M., et al. 1994. Hypoxia induces apoptosis with enhanced expression of fas antigen messenger RNA in cultured neonatal rat cardiomyocytes. Circ. Res. 75:426-433.

4. Bialik, S., et al. 1997. Myocyte apoptosis during acute myocardial infarction in the mouse localizes to hypoxic regions but occurs independently of p53. J. Clin. Invest. 100:1363-1372.

5. Gottlieb, R.A., Burleson, K.O., Kloner, R.A., Babior, B.M., and Engler, R.L. 1994. Reperfusion injury induces apoptosis in rabbit cardiomyocytes. J. Clin. Invest. 94:1621-1628.

6. Maulik, N., et al. 1999. Ischemic preconditioning reduces apoptosis by upregulating anti-death gene Bcl-2. Circulation. 100:II369-II375.

7. Webster, K.A., et al. 1999. Hypoxia-activated apoptosis of cardiac myocytes requires reoxygenation or a $\mathrm{pH}$ shift and is independent of p53. J. Clin. Invest. 104:239-252.

8. Kang, P.M., Haunstetter, A., Aoki, H., Usheva, A., and Izumo, S. 2000.
} 
Morphological and molecular characterization of adult cardiomyocyte apoptosis during hypoxia and reoxygenation. Circ. Res. 87:118-125.

9. Freude, B., et al. 2000. Apoptosis is initiated by myocardial ischemia and executed during reperfusion. J. Mol. Cell Cardiol. 32:197-208.

10. Anversa, P. 2000. Myocyte death in the pathological heart. Circ. Res. 86:121-124.

11. Elsasser, A., Suzuki, K., Lorenz-Meyer, S., Bode, C., and Schaper, J. 2001. The role of apoptosis in myocardial ischemia: a critical appraisal. Basic Res. Cardiol. 96:219-226.

12. Black, S.C., et al. 1998. Co-localization of the cysteine protease caspase-3 with apoptotic myocytes after in vivo myocardial ischemia and reperfusion in the rat. J. Mol. Cell. Cardiol. 30:733-742.

13. Bialik, S., et al. 1999. The mitochondrial apoptotic pathway is activated by serum and glucose deprivation in cardiac myocytes. Circ. Res. 85:403-414.

14. Jeremias, I., et al. 2000. Involvement of CD95/Apo1/Fas in cell death after myocardial ischemia. Circulation. 102:915-920.

15. de Moissac, D., Gurevich, R.M., Zheng, H., Singal, P.K., and Kirshenbaum, L.A. 2000. Caspase activation and mitochondrial cytochrome C release during hypoxia-mediated apoptosis of adult ventricular myocytes. J. Mol. Cell Cardiol. 32:53-63.

16. Chen, M., et al. 2001. Bid is cleaved bycalpain to an active fragment in vitro and during myocardial ischemia/reperfusion. J. Biol. Chem. 276:30724-30728.

17. Reed, J.C., and Paternostro, G. 1999. Postmitochondrial regulation of apoptosis during heart failure. Proc. Natl. Acad. Sci. U. S. A. 96:7614-7616.

18. Beltrami, A.P., et al. 2001. Evidence that human cardiac myocytes divide after myocardial infarction. N. Engl. J. Med. 344:1750-1757.

19. Ma, X.L., et al. 1999. Inhibition of p38 mitogen-activated protein kinase decreases cardiomyocyte apoptosis and improves cardiac function after myocardial ischemia and reperfusion. Circulation. 99:1685-1691.

20. Mackay, K., and Mochly-Rosen, D. 1999. An inhibitor of p38 mitogenactivated protein kinase protects neonatal cardiac myocytes from ischemia. J. Biol. Chem. 274:6272-6279.

21. Dougherty, C.J., et al. 2002. Activation of c-Jun N-terminal kinase promotes survival of cardiac myocytes after oxidative stress. Biochem. J. 362:561-571.

22. Aoki, H., et al. 2002. Direct activation of mitochondrial apoptosis machinery by c-Jun $\mathrm{N}$-terminal kinase in adult cardiac myocytes. J. Biol. Chem. 277:10244-10250.

23. Bishopric, N.H., Andreka, P., Slepak, T., and Webster, K.A. 2001. Molecular mechanisms of apoptosis in the cardiac myocyte. Curr. Opin. Pharmacol. 1:141-150.

24. Hreniuk, D., et al. 2001. Inhibition of c-Jun N-terminal kinase 1, but not c-Jun N-terminal kinase 2, suppresses apoptosis induced by ischemia/reoxygenation in rat cardiac myocytes. Mol. Pharmacol. 59:867-874.

25. Creasy, C.L., and Chernoff, J. 1995. Cloning and characterization of a human protein kinase with homology to Ste20. J. Biol. Chem. 270:21695-21700.

26. Taylor, L.K., Wang, H.C., and Erikson, R.L. 1996. Newly identified stressresponsive protein kinases, Krs-1 and Krs-2. Proc. Natl. Acad. Sci. U. S. A. 93:10099-10104.

27. Kyriakis, J.M. 1999. Signaling by the germinal center kinase family of protein kinases. J. Biol. Chem. 274:5259-5262.

28. Dan, I., Watanabe, N.M., and Kusumi, A. 2001. The Ste20 group kinases as regulators of MAP kinase cascades. Trends Cell Biol. 11:220-230.

29. Lee, K.K., et al. 1998. Proteolytic activation of MST/Krs, STE20-related protein kinase, by caspase during apoptosis. Oncogene. 16:3029-3037.
30. Kakeya, H., Onose, R., and Osada, H. 1999. Activation of a 36-kD MBP kinase, an active proteolytic fragment of MST/Krs proteins, during anticancer drug-induced apoptosis. Ann. N. Y. Acad. Sci. 886:273-275.

31. Graves, J.D., et al. 1998. Caspase-mediated activation and induction of apoptosis by the mammalian STE-20 like kinase Mst1. EMBO J. 8:2224-2234.

32. Lee, K.K., Ohyama, T., Yajima, N., Tsubuki, S., and Yonehara, S. 2001. Mst, a physiological caspase substrate, highly sensitizes apoptosis both upstream and downstream of caspase activation. J. Biol. Chem. 276:19276-19285.

33. Ura, S., Masuyama, N., Graves, J.D., and Gotoh, Y. 2001. MST1-JNK promotes apoptosis via caspase-dependent and independent pathways. Genes Cells. 6:519-530.

34. Aoki, H., Izumo, S., and Sadoshima, J. 1998. Angiotensin II activates RhoA in cardiac myocytes:a critical role of RhoA in angiotensin IIinduced premyofibril formation. Circ. Res. 81:666-676.

35. Yamamoto, S., Seta, K., Morisco, C., Vatner, S., and Sadoshima, J. 2001. Chelerythrine rapidly induces apoptosis through generation of reactive oxygen species in cardiac myocytes. J. Mol. Cell. Cardiol. 33:1829-1848.

36. Geng, Y.J., et al. 1999. Apoptosis of cardiac myocytes in Gsalpha transgenic mice. Circ. Res. 84:34-42.

37. Yamamoto, S., Seta, K., Morisco, C., Vatner, S.F., and Sadoshima, J. 2001. Chelerythrine rapidly induces apoptosis through generation of reactive oxygen species in cardiac myocytes. J. Mol. Cell Cardiol. 33:1829-1848.

38. Sadoshima, J., et al. 2002. The MEKK1-JNK pathway plays a protective role in pressure overload, but does not mediate cardiac hypertrophy. J. Clin. Invest. 110:271-279. doi:10.1172/JCI200214938.

39. Sadoshima, J., Qiu, Z., Morgan, J.P., and Izumo, S. 1995. Angiotensin II and other hypertrophic stimuli mediated by $\mathrm{G}$ protein-coupled receptors activate tyrosine kinase, mitogen-activated protein kinase, and 90-kD S6 kinase in cardiac myocytes: the critical role of $\mathrm{Ca}^{2+}$-dependent signaling. Circ. Res. 76:1-15.

40. Yang, G., et al. 2001. Cyclosporine reduces left ventricular mass with chronic aortic banding in mice, which could be due to apoptosis and fibrosis. J. Mol. Cell. Cardiol. 33:1505-1514.

41. Gundersen, H.J.G. 1977. Notes on the estimation of the numerical density of arbitrary profiles: the edge effect. J. Microsc. 111:219-223.

42. Olivetti, G., Capasso, J.M., Sonnenblick, E.H., and Anversa, P. 1990. Sideto-side slippage of myocytes participates in ventricular wall remodeling acutely after myocardial infarction in rats. Circ. Res. 67:23-34.

43. Kim, S.J., et al. 1999. Differential regulation of inotropy and lusitropy in overexpressed Gsalpha myocytes through cAMP and $\mathrm{Ca}^{2+}$ channel pathways. J. Clin. Invest. 103:1089-1097.

44. Masaki, H., Sato, Y., Luo, W., Kranias, E.G., and Yatani, A. 1997. Phospholamban deficiency alters inactivation kinetics of L-type $\mathrm{Ca}^{2+}$ channels in mouse ventricular myocytes. Am. J. Physiol. 272:H606-H612.

45. Lee, K.K., and Yonehara, S. 2002. Phosphorylation and dimerization regulate nucleocytoplasmic shuttling of mammalian STE20-like kinase (MST). J. Biol. Chem. 277:12351-12358.

46. Brewis, N., et al. 2000. Dilated cardiomyopathy in transgenic mice expressing a mutant A subunit of protein phosphatase 2A. Am. J. Physiol. Heart Circ. Physiol. 279:H1307-H1318.

47. Olivetti, G., et al. 1997. Apoptosis in the failing human heart. N. Engl. J. Med. 336:1131-1141.

48. Lin, Y., Khokhlatchev, A., Figeys, D., and Avruch, J. 2002. Death-associated protein 4 binds MST1 and augments MST1-induced apoptosis. J. Biol. Chem. 277:47991-48001.

49. Khokhlatchev, A., et al. 2002. Identification of a novel ras-regulated proapoptotic pathway. Curr. Biol. 12:253-265. 